\title{
Melorheostosis of the hand affecting the c6 sclerotome and presenting with carpal tunnel syndrome
}

\author{
Shalimar Abdullah ${ }^{1}$, MBBs, Ms, Noreen Fazlina Mat Nor ${ }^{1}$, MD, Nor Hazla Mohamed $\underline{H a f l a h}^{1}$, MBChB, MS
}

\begin{abstract}
Melorheostosis is a rare, progressive bone disease accompanied by hyperostosis and soft tissue fibrosis. While affected adults present with contracture and pain, children present with limb length discrepancy and deformity. We report the case of a 20-year-old woman with melorheostosis since childhood who presented with right hand deformity and numbness. Radiographs showed not only a combination of dense sclerosis and opacities, but also the classic 'flowing candle wax' appearance. Radiography can be used to identify melorheostosis, thus preventing unnecessary bone biopsies. Carpal tunnel release revealed the presence of a thickened flexor retinaculum and a degenerated median nerve distal to the retinaculum, but did not show hyperostosis. This case highlights the role of nerve decompression in melorheostosis and the importance of early identification of the disease to prevent unnecessary bone biopsies.
\end{abstract}

Keywords: biopsies, carpal tunnel syndrome, hand, melorheostosis

\section{INTRODUCTION}

Melorheostosis involves the bony periosteum, endosteum, and soft tissue (i.e. skin and subcutaneous tissue). It is an uncommon disease, with an incidence of 0.9 cases per million; hand involvement has only been reported sporadically. ${ }^{(1,2)}$ The symptoms are usually indefinite and vague, leading physicians to perform radiological examination of the patient's skeleton.

\section{CASE REPORT}

A 20-year-old female Asian university student presented with numbness and progressive deformity of her right hand. She was born with a mild flexion deformity of her right thumb, which progressively worsened. The condition had worsened considerably by the time the patient was six years old, at which treatment was finally sought. Treatment at a regional hospital included an attempt to straighten the patient's thumb using a wire, but the surgery failed and the deformity deteriorated further. Additionally, the patient's index and middle fingers began to exhibit limited flexion. The patient then defaulted on follow-up.

Numbness of the patient's thumb, and index and middle fingers started to develop at 13 years of age. She presented to a regional hospital different than that aforementioned, where the attending team ordered two bone biopsies of the humerus and radius, as they were unable to identify the disease. The subsequent report confirmed melorheostosis. There was no further treatment. By 16 years of age, the patient's right hand had a very limited range of motion, with clumsy movements and an inability to carry heavy items. She then began using her left hand for daily activities. At the age of 20 years, the patient sought treatment at our specialist centre due to the unbearable numbness. The patient also had associated pain in the right forearm and radial side of the hand, which was relieved by oral analgesics.

On examination, the most striking feature was the hypoplastic right hand with an atrophic thenar muscle. The right thumb was tightly adducted and had a fixed flexion of $60^{\circ}$ at the interphalangeal joint (Fig. 1). The index and middle fingers on the right hand were flexed at the proximal interphalangeal joint (PIPJ) and distal interphalangeal joint (DIPJ). The passive flexion of both the right index and middle fingers was found to be reduced at the metacarpophalangeal joint $\left(0^{\circ}-70^{\circ}\right)$, PIPJ $\left(30^{\circ}-50^{\circ}\right)$ and DIPJ $\left(20^{\circ}-70^{\circ}\right)$.

The range of movement of the right forearm and wrist was also limited with respect to supination $\left(45^{\circ}\right)$, pronation $\left(70^{\circ}\right)$, and wrist dorsiflexion $\left(0^{\circ}-20^{\circ}\right)$. Sensation was also impaired. Two-point discrimination test performed revealed a two-point threshold of $6 \mathrm{~mm}$ in both the radial and ulnar sides of the right thumb, and more than $20 \mathrm{~mm}$ in the radial and ulnar sides of index and middle finger. The patient's left hand revealed a two-point threshold of $5 \mathrm{~mm}$. Grip strength of the patient's right hand was unrecordable, while her left hand had a grip strength of 10 pounds. Although Tinel's test was negative at the patient's right carpal tunnel, Durkin's provocative test and Phalen's test of the right wrist were both positive. Both shoulders and elbows had full range of motion and were supple.

Radiographs of the right hand showed a well-circumscribed, dense sclerotic appearance of the patient's phalanges and metacarpal bones of the thumb and index finger (Fig. 2). Opacities and sclerotic areas were also seen in the scaphoid, lunate bone, trapezium, trapezoid, capitate, distal radius and the supracondylar processes of the bilateral humeri. Radiographs

${ }^{1}$ Department of Orthopaedics and Traumatology, Faculty of Medicine, Universiti Kebangsaan Malaysia, Kuala Lumpur, Malaysia

Correspondence: A/Prof Nor Hazla Mohamed Haflah, Consultant Orthopaedic Surgeon, Department of Orthopaedics and Traumatology, Faculty of Medicine, Universiti Kebangsaan Malaysia, Jalan Yaacob Latiff, Cheras, 56000 Kuala Lumpur, Malaysia. hazla1971@yahoo.com 

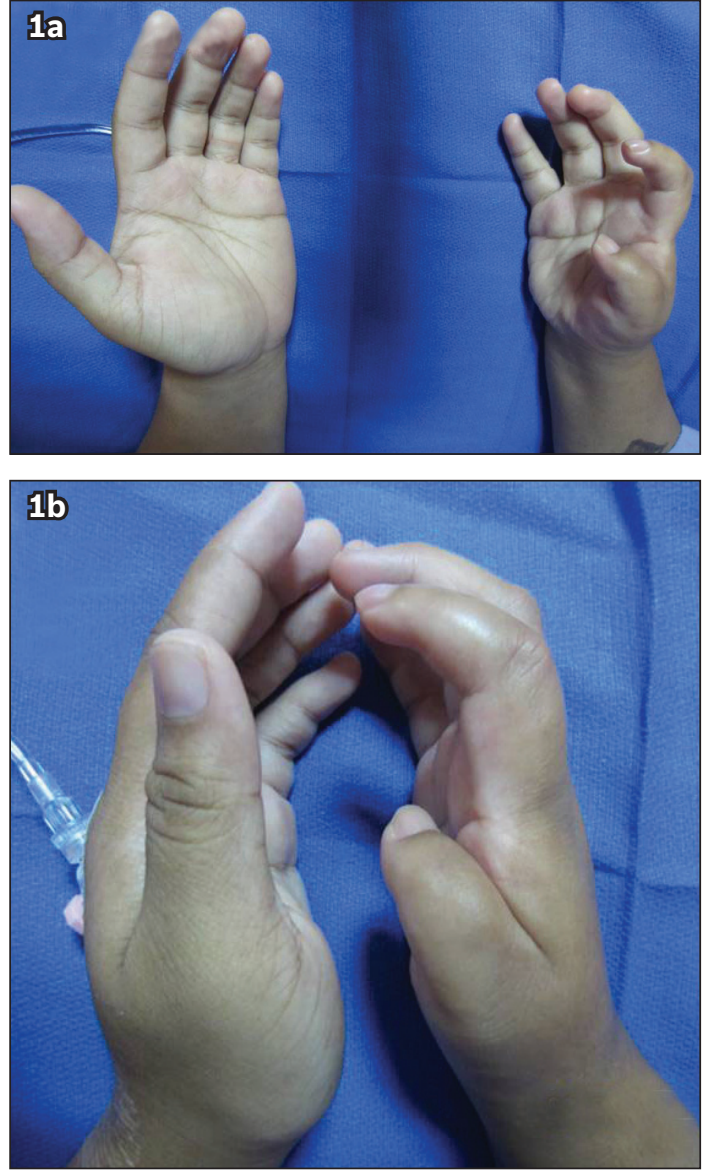

Fig. 1 Photographs comparing both hands of the patient show (a) a smaller right hand with flexion deformity of the right thumb, and index and middle fingers, as well as a shiny, tapering appearance of the index finger; and (b) gross atrophy of the right thenar muscles.

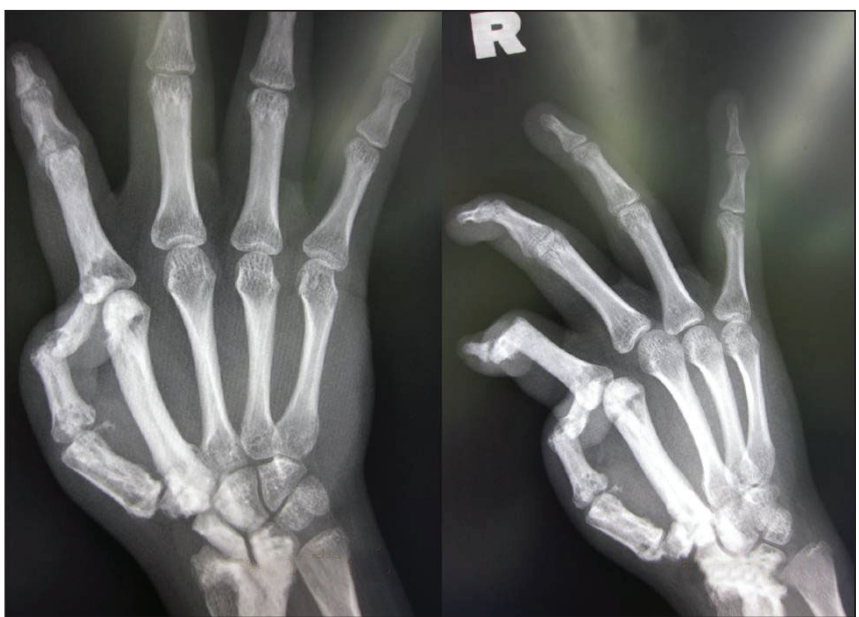

Fig. 2 Radiographs show a well-circumscribed, dense sclerotic appearance of the phalanges and metacarpal bones of the thumb and index finger. Sclerotic areas and opacities are present within the scaphoid, lunate, trapezium, trapezoid, capitates, distal radius and the supracondylar processes of the bilateral humeri.

of the left humerus showed the classic 'flowing candle wax' appearance, with sclerotic regions in the radius, carpals, metacarpals and phalanges. Magnetic resonance (MR) imaging of the right wrist revealed thickened bony cortex of the distal radius, ulna, scaphoid, lunate, trapezoid, trapezium, capitate, the first and second metacarpals, and the phalangeal bones,

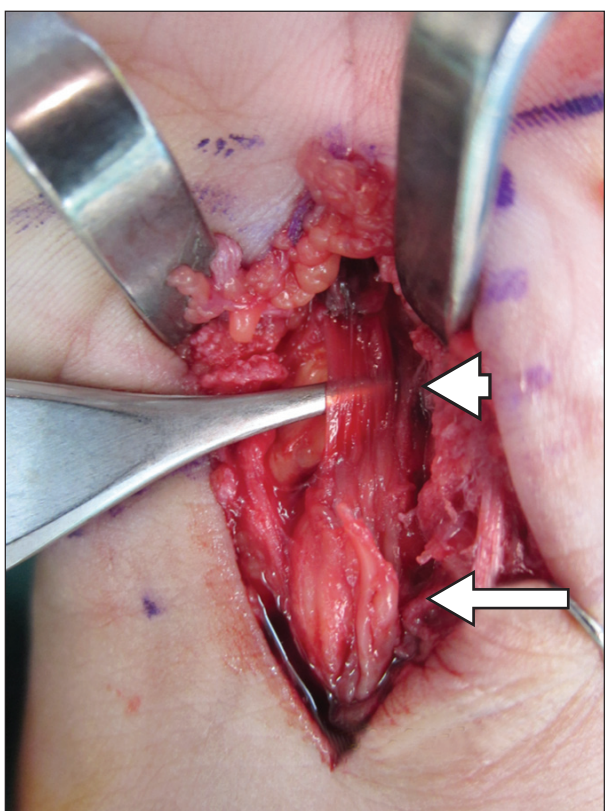

Fig. 3 Intraoperative photograph shows the median nerve. As the bulbous nerve at the proximal area (arrow) progresses distally, there is abrupt paper-thin flattening of the nerve (arrowhead). The paper-thin nerve, containing nerve fascicles, is elevated using a pair of forceps.

which were hypointense on T1- and T2-weighted MR imaging. Hypointensity on T1- and T2-weighted MR imaging is consistent with hyperostosis. The carpal tunnel was found to be narrowed, but no bony growth was seen protruding into the tunnel. Nerve conduction study revealed the absence of the sensory component of the right median nerve. We subsequently proceeded with an exploration of the patient's carpal tunnel. Intraoperatively, no hyperostosis of the carpal bones suggesting nerve compression was seen. However, the flexor retinaculum was found to be thickened and tight, compressing the median nerve and causing it to bulge proximal to the flexor retinaculum, as well as be flattened out paper-thin underneath the flexor retinaculum and distally (Fig. 3). Our findings were explained to the patient. She was discharged well, and commenced physiotherapy as an outpatient. At the six-month follow-up, the patient's numbness had reduced by about half.

\section{DISCUSSION}

Melorheostosis follows a sclerotomal distribution, and may be segmental or unilateral (hemimelic), affecting one bone (monostotic), one limb (monomelic) or multiple bones (polyostotic). ${ }^{(1)}$ Segmental sensory lesion occurs due to specific infection, insult or injury to a segment or segments of the neural crest during embryogenesis. In our patient, the C6 sclerotome was involved. If melorheostosis presents with the classical description of flowing cortical hyperostosis along the shaft of the long bone, resembling melting wax flowing down the side of a candle, simple clinical radiography and evaluation are usually adequate for the diagnosis of the condition. ${ }^{(1,2)}$ The present case involved a degenerated median nerve, possibly due to longstanding compression by the patient's tight flexor 
retinaculum. The atrophic median nerve likely resulted in the progressive deformity of her right hand, which was initially neglected, resulting in poor thumb opposition function and pain.

There are five other radiological presentations that resemble melorheostosis: (a) osteoma-like (30\%); (b) classic 'flowing candle wax' (22\%); (c) myositis ossificans-like (4\%); (d) osteopathia striata-like (26\%); and (e) mixed (18\%). ${ }^{(3)}$ The classic 'flowing candle wax' appearance was present in our patient's humerus; this should have allowed for the identification of melorheostosis, preventing two unnecessary bone biopsies. Abdullah et al reported two incidences of junior physicians misdiagnosing melorheostosis as possible malignancies, which resulted in the ordering of unnecessary bone biopsies. $^{(4)}$

A possible cause of nerve impingement in melorheostosis is either hyperostosis or tissue fibrosis. Although there was no hyperostosis in our patient, the carpal tunnel was found to be narrowed on MR imaging, with a thickened flexor retinaculum. Melorheostosis not only affects bones but also soft tissues, and could possibly result in a thick retinaculum. Symptoms of carpal tunnel syndrome are often seen in patients with melorheostosis, especially when the C6 sclerotome is involved, as was the case in our patient. (5) As only the right C6 sclerotome was affected in our patient, melorheostosis did not affect the patient bilaterally.

Pruitt and Manske ${ }^{(6)}$ reported two similar cases - both patients had contractures of the thumb and index finger, with pain and loss of function. Carpal tunnel release was performed on both patients to alleviate pain and paraesthesia. Concluding that involvement of the radial side of the hand is disabling due to the loss of thumb apposition, Pruitt and Manske suggested training the unaffected extremity in a dominant fashion as early as possible. ${ }^{(6)}$ In our patient's case, she had begun using her left hand more frequently from a young age upon realising that her right hand functioned poorly.

Our patient's median nerve had degenerated substantially distal to the thick flexor retinaculum, leaving only a paper-thin segment of continuous fascicles. We felt that a nerve graft was not indicated in our patient since these fascicles were continuous, despite Barfred and Ipsen having reported successfully performing such a graft in a six-year-old child with a degenerated median nerve. ${ }^{(7)}$

Soft tissue contracture can be progressive, causing greater deformity and disability than melorheostosis. Deformity of the affected limb and limb length discrepancy are caused by premature closure of the growth plate; fibrotic changes in the skin and soft tissue results in continued tethering as they do not stretch with the growth of the limb. ${ }^{(8-10)}$ Due to loss of thumb apposition, involvement of the radial aspect of the hand is disabling. ${ }^{(6)}$ In our patient, we offered a further operation to fuse her thumb in an abducted position for better functionality.

As melorheostosis is a progressive disease, its signs may not be obvious during childhood. Initial problems include limb deformity and limb length discrepancy, which are later followed by the development of progressive deformity, contractures, and pain with possible nerve compression. The classic 'flowing candle wax' appearance should enable identification of melorheostosis without the need for bone biopsies. We strongly suggest performing early carpal tunnel release upon the onset of symptoms, in order to avoid further degeneration of the nerve.

\section{REFERENCES}

1. Suresh S, Muthukumar T, Saifuddin A. Classical and unusual imaging appearances of melorheostosis. Clin Radiol 2010; 65:593-600.

2. Ameen S, Nagy L, Gerich U, Anderson SE. Melorheostosis of the hand with complicating bony spur formation and bursal inflammation: diagnosis and treatment. Skeletal Radiol 2002; 31:467-70.

3. Freyschmidt J. Melorheostosis: a review of 23 cases. Eur Radiol 2001; 11:474-9.

4. Abdullah S, Pang GM, Mohamed-Haflah NH, Sapuan J. Melorheostosis of the ulna. J Chin Med Assoc 2011; 74:469-72.

5. Landi A, Leti Acciaro A, Della Rosa N, Pellacani A. Carpal Tunnel Syndrome: Rare causes. Berlin Heidelberg: Springer, 2007: 95-100.

6. Pruitt DL, Manske PR. Soft tissue contractures from melorheostosis involving the upper extremity. J Hand Surg Am 1992; 17:90-3.

7. Barfred T, Ipsen T. Congenital carpal tunnel syndrome. J Hand Surg 1985; 10:246-8.

8. Campbell CJ, Papademetriou T, Bonfiglio M. Melorheostosis. A report of the clinical, roentgenographic, and pathological findings in fourteen cases. J Bone Joint Surg Am 1968; 50:1281-304.

9. Rozencwaig R, Wilson MR, McFarland GB JR. Melorheostosis of the skeletally immature hand: a case report and long term follow-up evaluation. J Hand Surg Am 1996; 21:703-6.

10. Younge D, Drummond D, Herring J, Cruss RL. Melorheostosis in children. Clinical features and natural history. J Bone Joint Surg $\mathrm{Br}$ 1979; 61-B:415-8. 